\title{
Women's awareness of the importance of long-chain omega-3 polyunsaturated fatty acid consumption during pregnancy: knowledge of risks, benefits and information accessibility
}

\author{
Danka S Sinikovic ${ }^{1}$, Heather R Yeatman ${ }^{1}$, Deborah Cameron ${ }^{3}$ and Barbara J Meyer ${ }^{1,2, *}$

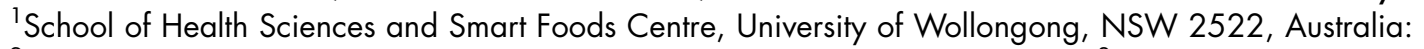 \\ ${ }^{2}$ Metabolic Research Centre, University of Wollongong, NSW 2522, Australia: ${ }^{3}$ Northern Illawarra Maternal \\ and Paediatric Services, Illawarra Area Health Service, NSW 2500, Australia
}

Submitted 13 August 2007: Accepted 20 March 2008: First published online 29 May 2008

\begin{abstract}
Objective: To evaluate pregnant women's knowledge regarding the importance of long-chain omega-3 polyunsaturated fatty acids (LC $n-3$ PUFA) consumption during pregnancy and assess their views on current information availability.

Design: A 27-item demographic and food safety/behaviour questionnaire was administered to pregnant women during their antenatal clinic visits. $\chi^{2}$ tests were performed using SPSS.

Setting: Antenatal clinics at two regional hospitals in New South Wales, Australia. Subjects: One hundred and ninety ( $n$ 190) pregnant women.

Results: Three quarters of the women had not received information regarding LC $n$-3 PUFA. Approximately half of the women were aware of issues relating to LC $n$-3 PUFA; however, their knowledge was limited, with most obtaining their knowledge from books and magazines. Women generally had low (30\%, 29\%) to moderate $(28 \%, 24 \%)$ levels of concern about LC $n-3$ PUFA and mercury, respectively.

Conclusions: Pregnant women lack knowledge of LC $n$-3 PUFA and health-care services do not provide pregnant women with adequate information on the importance of eating foods high in LC $n-3$ PUFA during pregnancy.
\end{abstract}

Keywords
Pregnant women's knowledge
Omega-3 polyunsaturated fatty acids
Pregnancy
Food safety
Risks and benefits
Nutrition

Omega-3 polyunsaturated fatty acids ( $n$-3 PUFA) are important for health, including cardiovascular health ${ }^{(1)}$, mental health ${ }^{(2)}$ and inflammatory disorders ${ }^{(3)}$. Most of these health benefits have been attributed to the longchain (LC) eicosapentaenoic acid (EPA) and docosahexaenoic acid (DHA).

LC $n$-3 PUFA can be synthesised from $\alpha$-linolenic acid but the conversion through to DHA is limited ${ }^{(4)}$. Hence, direct consumption of DHA is preferred. The main dietary source of DHA is fish and seafood ${ }^{(5,6)}$. DHA is a major structural component membrane phospholipids in utero and in infancy, especially of the nervous system ${ }^{(7)}$. Due to the importance of DHA in the brain and retina, it plays a crucial role in normal neurotransmission and visual function $^{(8,9)}$, as well as in controlling the processes of learning and development ${ }^{(10)}$. Decreased levels of DHA have been associated with depression ${ }^{(11,12)}$ and trials in assessing the effects of DHA supplementation in postnatal depression are currently underway.

During pregnancy there are many metabolic changes that occur, including the mobilisation of nutrients for the growing fetus. One such nutrient that is mobilised during pregnancy is DHA, which is crucial for proper neural, visual and cognitive development of the fetus ${ }^{(9,11)}$. Al et al. showed that DHA and arachidonic acid are both transferred to the fetus during pregnancy ${ }^{(13)}$. Arachidonic acid is easily synthesised from linoleic acid, which is abundant in the diet, whereas DHA is consumed in much lower amounts ${ }^{(5,6)}$. If the maternal intake of DHA is limiting, DHA will be mobilised from the maternal stores to supply the growing fetus with this essential nutrient $^{(13)}$.

The National Health and Medical Research Council (NHMRC) has released the Nutrient Reference Values for Australia and New Zealand and recommends LC $n-3$ PUFA intake of $160 \mathrm{mg} / \mathrm{d}$ for adult men and $90 \mathrm{mg} / \mathrm{d}$ for adult women ${ }^{(14)}$. For pregnancy, the recommendation is higher at $110 \mathrm{mg} / \mathrm{d}$ for $14-18$-year-olds and $115 \mathrm{mg} / \mathrm{d}$ for $19-50$-year-olds ${ }^{(14)}$. However, the International Society for the Study of Fatty Acids and Lipids (ISSFAL) recommends $300 \mathrm{mg}$ of DHA daily for pregnant women $^{(15)}$. Currently, Australian women (19 years and older) are consuming $195 \mathrm{mg}$ LC $n$-3 PUFA daily comprising of $60 \mathrm{mg} \mathrm{EPA}, 52 \mathrm{mg}$ docosapentaenoic acid 
and $83 \mathrm{mg} \mathrm{DHA}^{(6)}$. These current intakes meet the NHMRC recommendations but fall short of the ISSFAL recommendations. Insufficient intakes of DHA have particularly been noted for the developed nations such as Australia, Canada, the UK and the USA ${ }^{(16)}$.

Pregnant women and their babies are a priority public health target group, making this group the main target in education and promotion of the positive effects of LC $n-3$ PUFA, including DHA. Although fish and seafood are the richest source of DHA, they can be a major source of contaminants such as methyl mercury, polychlorinated biphenyls, dioxins and other environmental contaminants ${ }^{(17)}$. Larger and longer living predatory fish, such as shark, ray, swordfish and gemfish, accumulate higher levels of mercury and it is these fish that pose the greatest threat of exposure to mercury, but the smaller fish such as shellfish, salmon and canned (or fresh) tuna accumulate very low levels of mercury ${ }^{(18)}$. The areas most affected by higher levels of mercury accumulation in fish are those that develop in acidic waters ${ }^{(19)}$. Methyl mercury, particularly, can diminish the effect of LC $n-3$ PUFA in fish, which can be harmful to the developing nervous system ${ }^{(17,20)}$. However, a recent study by Hibbeln et $a l .{ }^{(21)}$ found that women who consumed more than $340 \mathrm{~g}$ of fish/seafood per week (equivalent to two fish meals a week) during their pregnancy had children with higher IQ at age 8 compared with the children whose mothers consumed less than $340 \mathrm{~g}$ of fish/seafood per week and no detrimental effects of fish/seafood consumption were reported ${ }^{(21)}$.

Adverse messages and/or negative press about food health issues can influence people's decisions with respect to incorporation of fish in the diet. A study of consumer perception about fish consumption found that a relatively high proportion of subjects, $43 \%$ of a total 429 respondents, did not eat fish at least once a week due to negative perceptions on fish intake ${ }^{(22)}$. Pregnant women may lack familiarity with the 'right' foods, creating a major barrier to adequate LC $n-3$ PUFA intake ${ }^{(23)}$. Adequate amounts of information and accessibility of this information, as well as guidelines, will assist women to gain the benefits of eating fish rich in LC $n-3$ PUFA and would outweigh the risks, ensuring they and their babies gain maximum nutritional benefits ${ }^{(24)}$. Fortunately, research has acknowledged that Australian seafood is the best source of omega-3 oils and that it is safe to consume either wild or farmed seafood ${ }^{(25)}$.

Pregnant women actively seek advice about various health-related topics, including nutritional changes, to ensure the healthy development of their baby ${ }^{(26)}$. Nutrition information routinely provided to pregnant women has a focus on nutrients, predominantly calcium, iron and folate ${ }^{(27-29)}$. Recommendations on the provision of this information have been put into place, and sufficient information is available to women regarding the need to increase consumption of foods high in these nutrients ${ }^{(28)}$.
However, less information is available regarding the importance of LC $n-3$ PUFA and the safety issues associated with intake of fish during pregnancy ${ }^{(11,26,27,30,31)}$.

No study has been reported that examines pregnant women's awareness, knowledge and views on current information availability regarding LC $n-3$ PUFA. Assessment of communication strategies between health-care providers and pregnant women in relation to LC $n-3$ PUFA is an important step in the development of effective nutrition education strategies.

The aims of the study were: (i) to provide an insight into pregnant women's knowledge of LC $n-3$ PUFA; (ii) to assess their understanding of the potential risks associated with the consumption of fish/seafood during pregnancy; and (iii) to assess their views on current information availability in relation to the importance of LC $n-3$ PUFA and their safe consumption during pregnancy.

\section{Methods}

\section{Development and pilot testing of survey}

The survey questions were constructed on the basis of information obtained from interviews with health-care providers, published elsewhere ${ }^{(32)}$. Questions explored the extent to which information from health-care providers reached pregnant women and assessed the pregnant women's knowledge of LC $n-3$ PUFA. The survey was divided into three sections in relation to the question content: (i) demographics; (ii) food safety attitude/behaviour; and (iii) LC $n-3$ PUFA/mercury. The demographic questions included education levels, income levels and the number of weeks pregnant. The food safety attitude/ behaviour section involved questions on sources of information utilised by the women and the specific (nutrition) information they sought. Specific LC $n-3$ PUFA/mercury questions involved knowledge of LC $n-3$ PUFA and main concerns during pregnancy associated with fish consumption.

A 26-item questionnaire was trialled in a pilot study ( $n$ 10). Quality control checks were performed during the course of the pilot to ensure a robust instrument was developed and an additional question was added. The questionnaire was reviewed for content validity by researchers with expertise in nutrition/food safety survey development. This aimed to minimise risks associated with possible anxiety experiences sometimes associated with food safety questionnaires. Two groups of five women assisted with minor editing and question sequencing modifications to improve the flow and clarity of the questionnaire.

\section{Subject recruitment}

Pregnant women were recruited to participate in completing a self-administered questionnaire while waiting for their appointment at antenatal clinics at two regional 
hospitals in NSW, Australia, one of which was a coastal and the other an inland hospital. The hospitals were selected based on convenience in terms of geographic location and accessibility. Information sheets and flyers were used to recruit participants during the times of the clinics.

\section{Data collection}

Pregnant women who attended antenatal clinics were approached to participate in the study. The 27-item survey was self-administered by the majority of women, although, if necessary, the women could ask the researcher for assistance. All women were informed about the purpose of the study and written consent was obtained prior to completing the survey. Procedures were approved by the Human Ethics Research Committee of University of Wollongong. Duration of participation ranged from 10-15 min.

\section{Data analysis}

Data were coded and analysed using SPSS (Statistical Package for Social Sciences) version 13.0 for Windows (SPSS Inc., Chicago, IL, USA). Open-ended responses were coded for analysis. $\chi^{2}$ testing was used to determine statistically significant relationships within the data, with a level of significance set at $\alpha=0.05$ for all analyses.

\section{Results}

\section{Subject characteristics}

A sample of 190 women was recruited from two hospital antenatal clinics (Table 1). The majority of the women $(67 \%)$ surveyed were in the third trimester of their pregnancy. For most of the women this was not their first pregnancy (66\%) and, of these, $62 \%$ were in their second pregnancy. For $64 \%$ of the participants, the current pregnancy was planned. Approximately one-third of women in this study have obtained tertiary qualifications (27\%), one-third have completed high school (31\%) and $40 \%$ of women have completed TAFE (Technical and Further Education/community college). Similarly, onethird of the women's income was no greater than $\$ 20000$, one-third of the women had an income between $\$ 20001$ and $\$ 40000$, and approximately one-third had an annual income between $\$ 40001$ and $\$ 75001$. Surveys classified the type of antenatal care the women were receiving in two ways: 'Antenatal Shared Care Programme' (women are under the care of their family doctor in conjunction with midwives at antenatal clinics) and 'Midwife Group Practice' (women predominantly under the care of midwives at antenatal clinics). Two-thirds of the women were involved in the Antenatal Shared Care Programme (67\%) for their antenatal care.

\section{Receipt of LC n-3 PUFA information}

Only approximately one-quarter of women (23\%) received information about LC $n$-3 PUFA (Fig. 1). Similarly,
Table 1 Characteristics of pregnant women ( $n$ 190)

\begin{tabular}{lrr}
\hline Characteristic & $n$ & $\%$ \\
\hline Stage of pregnancy & & \\
$\quad$ First trimester & 18 & 10 \\
Second trimester & 44 & 23 \\
$\quad$ Third trimester & 128 & 67 \\
Number of pregnancies & & \\
One & 64 & 34 \\
Two & 78 & 41 \\
Three & 36 & 19 \\
Four or more & 12 & 6 \\
Planned pregnancy & 121 & 64 \\
Yes & 69 & 36 \\
No & & \\
Income (\$ per year) & 24 & 13 \\
<10 000 & 31 & 16 \\
10 001-20 000 & 60 & 32 \\
20 001-40 000 & 54 & 28 \\
40 001-75 000 & 21 & 11 \\
$>75000$ & & \\
Education & 3 & 2 \\
Primary & 59 & 31 \\
Secondary & 75 & 39 \\
TAFE & & 11 \\
University undergraduate & 20 & 16 \\
University postgraduate & 21 \\
Othert & & \\
Form of care & & \\
Antenatal Shared Care Programme & 127 & 67 \\
Midwife Group Practice & 63 & 33 \\
\hline
\end{tabular}

*TAFE: Technical and Further Education (community college). tOther: college diploma, university certificate.

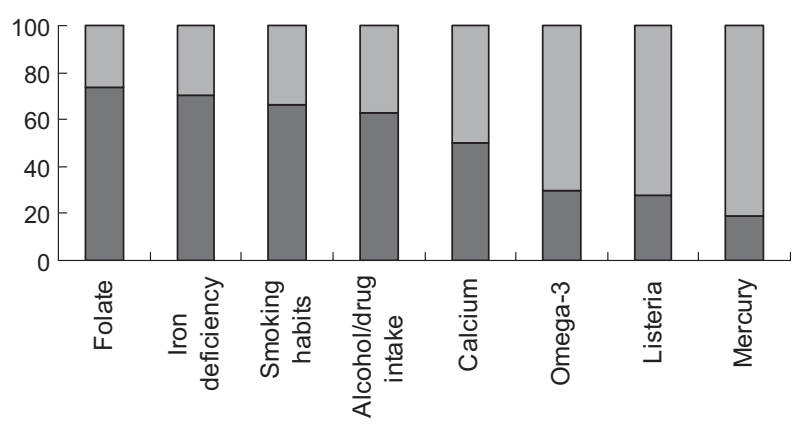

Fig. 1 Issues discussed with women during pregnancy. $\square$ Yes, issue has been raised with pregnant woman; $\square$ No, issue had not been raised with pregnant woman.

only a small number of women reported antenatal discussions about mercury (19\%) and listeria during pregnancy $(27 \%)$. Other health and nutrition topics reported to be frequently discussed by the health-care providers with pregnant women included smoking habits, iron deficiency, alcohol/drug intake, folate and calcium (66\%, $71 \%, 63 \%, 74 \%$ and 50\%, respectively) (Fig. 1). Even though a larger proportion of information provided to women during the course of their pregnancy was on food and nutrition changes (35\%), labour/birth was another frequently discussed topic area (33\%). Similarly, the information pregnant women generally inquired about 
were food and nutrition changes (34\%) and/or labour/ birth (33\%).

\section{Knowledge about LC n-3 PUFA}

Despite a high number of women having received no information about LC $n-3$ PUFA, more than half of the women (55\%) were aware of issues regarding risks and benefits of eating fish during pregnancy, while $20 \%$ of women answered 'not sure' and $25 \%$ reported they were not aware at all. The women who were aware of issues related to eating fish during pregnancy were asked to identify the issues. Most knowledge related to risk associated with mercury (37\%) and benefits of LC $n$-3 PUFA (29\%).

Women who had not received information about LC $n$-3 PUFA during pregnancy ( $n$ 147) reported lower awareness of issues related to risks and benefits of eating fish during this time as opposed to women who had received information on LC $n$-3 PUFA ( $n$ 43) (Pearson $\chi^{2}$ $12 \cdot 288, P=0 \cdot 002$ ).

The main message received by those women reporting having received anything ( $n$ 43) was related to baby's brain development $(56 \%)$, followed by non-specific benefits associated with LC $n$-3 PUFA (30\%). A minority of these women could not recall the importance of this fatty acid $(14 \%, n 6)$.

Of those women who were aware of issues related to eating fish ( $n$ 105), $70 \%$ were women who had planned their current pregnancy, showing a significant association (Pearson $\chi^{2} 7 \cdot 277, P=0 \cdot 026$ ). Having a prior pregnancy did not affect the level of awareness of LC $n-3$ PUFA and fish consumption (Pearson $\chi^{2} 1 \cdot 118, P=0 \cdot 891$ ).

The results show a significant association between women's level of income and awareness related to fish consumption (Pearson $\chi^{2} 16.733, P<0 \cdot 05$ ). Similarly, an association was found between level of education and knowledge about LC $n-3$ PUFA (Pearson $\chi^{2} 8 \cdot 165$, $P=0 \cdot 017)$. Women with higher levels of education were more aware of issues related to eating fish during pregnancy. Upon evaluating each of these independent variables by performing a multiple regression analysis, level of education remained statistically significant $(P=0 \cdot 012)$ and thus provides a stronger contribution to the level of awareness.

\section{Information sources}

The form of antenatal care received by the women was not found to be associated with women's knowledge (Pearson $\chi^{2} 0 \cdot 817, P=0 \cdot 665$ ) or having received information about LC $n$-3 PUFA (Pearson $\chi^{2} 1 \cdot 021, P=0 \cdot 204$ ). In terms of initial contact after learning about their pregnancy, women most often saw their family doctors (59\%), family and friends combined (26\% and $13 \%$ each), followed by midwives ( $8 \%$ ). The family doctor was reported to be the main source of general information for pregnant women, and an association was found between source of information and level of income (Pearson $\chi^{2}$ 3.995, $P=0.035)$.

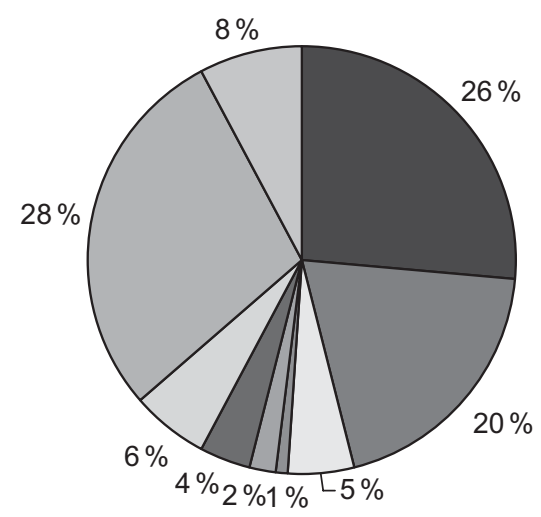

Fig. 2 Sources of information on LC $n-3$ PUFA ( $n$ 102). $\square$ Family doctor ( $n$ 27), $\square$ midwife $(n 20), \square$ dietitian $(n 5)$, $\square$ obstetrician ( $n 1)$, $\square$ family member $(n 2)$, $\square$ friend $(n 4)$, $\square$ Internet ( $n$ 6), $\square$ books/magazines ( $n$ 29) and $\square$ others, e.g. TV, IVF clinics ( $n$ 8)

Table 2 Channels of receiving information during pregnancy*

\begin{tabular}{lccccc}
\hline & $\begin{array}{c}\text { How women receive } \\
\text { information on LC } n \text {-3 } \\
\text { PUFA }(n \text { 43) }\end{array}$ & \multicolumn{2}{c}{$\begin{array}{c}\text { Preferred channel of } \\
\text { receiving information } \\
\text { on LC } n \text {-3 PUFA }(n \text { 55) }\end{array}$} \\
\cline { 2 - 3 } \cline { 5 - 6 } & $n$ & $\%$ & & $n$ & $\%$ \\
\hline Pamphlets & 29 & 48 & & 50 & 72 \\
In-person & 22 & 37 & & 10 & 14 \\
Education classes & 3 & 5 & & 8 & 11 \\
Other & 6 & 10 & & 2 & 3 \\
\hline
\end{tabular}

*Participants could have chosen more than one option.

With respect to LC $n$-3 PUFA, women received information from a wide range of sources, as presented in Fig. 2. Books and magazines were reported to be the most common source of information ( $28 \%$ ), followed by family doctors (26\%) and midwives (20\%). Significant associations were found between the tertiary level of education and accessing information about LC $n-3$ PUFA from books/magazines (Pearson $\chi^{2} 10 \cdot 311, P=0 \cdot 001$ ). Women with higher levels of education were more likely to seek out information themselves. Women with lower levels of education, i.e. 'primary and secondary', were less likely to receive information about LC $n-3$ PUFA (Pearson $\chi^{2}$ 4.529, $P=0 \cdot 024$ ) (Fig. 2).

Women who had received information about LC $n-3$ PUFA from a health-care provider identified pamphlets as the most common form of receiving that information (48\%) (Table 2). Many women also identified receiving information verbally (37\%). Women's preferred channel of receiving information was overwhelmingly identified as pamphlets (72\%).

\section{Need for more information and awareness of risks and benefits of fish consumption}

A majority of the women ( $n 137 ; 72 \%)$ stated they would not like to receive more information about LC $n$-3 PUFA 
and fish consumption. Furthermore, of those who had answered not having received information on fish consumption during pregnancy ( $n 147 ; 77 \%$ ), only forty-four women $(30 \%)$ would like to receive more information. No significant association was found between having received information and a need for more information (Pearson $\chi^{2} 0 \cdot 729, P=0 \cdot 257$ ).

Regarding awareness of safe fish consumption during pregnancy, 105 women were aware (55\%), forty-eight women were not aware (25\%) and thirty-seven women were not sure $(20 \%)$. Of the women who were not aware of any issues relating to fish risks or benefits ( $n 48 ; 25 \%$ ), a majority ( $n 33 ; 69 \%$ ) would not like to receive more information about LC $n-3$ PUFA and fish consumption. Of the women that had requested further information about LC $n$-3 PUFAs ( $n 53 ; 28 \%$ ), said they wanted 'any information' ( $n$ 22; $42 \%$ ), followed by 'why important' ( $n$ 7; $14 \%)$ and 'recommendations and benefits' ( $n$ 4; $7 \%$ each).

\section{Views on current information availability}

On the scale of concern from one to five across a range of topic areas, women rated LC $n-3$ PUFA and mercury mostly as 'very low' levels of concern, 30\% and 29\%, respectively. Approximately equal proportions of participants reported having 'moderate concern' about LC $n$-3 PUFA and mercury, $28 \%$ and $24 \%$, respectively. The smallest percentage of participants rated LC $n$-3 PUFA and mercury as 'very high' level of concern, $5 \%$ each. Levels of concern about LC $n$-3 PUFA were not associated with increasing number of pregnancies (Pearson $\chi^{2} 2 \cdot 158$, $P=0.905)$. A high proportion of women $(85 \%)$ had no concerns about eating fish regularly.

The issue of most concern that was raised among women was labour/birth. The least knowledge about a certain issue across all topic areas was listeria, rating highest for the 'not sure' category at $32 \%$.

\section{Discussion}

\section{Pregnant women's knowledge and risks and benefits of LC n-3 PUFA}

The present study identified that only a small number of women had received even limited information about LC $n$-3 PUFA and safe fish consumption from their healthcare provider. Women reported very limited exposure to information on the importance of LC $n-3$ PUFA and safe fish intake during pregnancy. In fact, the survey data showed a high proportion of women ( $77 \%$ ) receiving no information on these issues during their health-care visits. The survey data also indicated that many women would like 'any' information about LC $n$-3 PUFA and safe fish consumption, confirming that their knowledge was very limited.

Previous studies have identified a gap in the knowledge of food safety among women, indicating they may not be comprehending the message that consumption of some fish species during pregnancy can adversely affect their baby ${ }^{(31,33)}$. Athearn et al. found that women were not informed about and were unaware of potential risks of consuming certain foods during pregnancy ${ }^{(31)}$. Any potential risk to a baby can be an incentive for pregnant women to access suitable information ${ }^{(31)}$. This has important implications for education initiatives to promote food safety, specifically in terms of mercury in fish.

The study results indicated a high level of interest among the women about various nutrition and food safety matters that were important for the healthy development of their baby. In some instances this interest prompted information-seeking behaviours, particularly by women with higher levels of education. A previous study investigating information-seeking behaviour of pregnant women found that previous knowledge may influence search behaviours ${ }^{(34)}$. This may indicate that a programme to communicate LC $n$ - 3 PUFA and safe fish consumption information to the general population may raise an awareness of the issues and lead to the promotion of further information-seeking behaviours.

\section{Response to information received}

Women rated food and nutrition changes (in conjunction with labour/birth) as the most sought and provided information. However, LC n-3 PUFA and safe fish consumption information did not appear to be communicated regularly as part of the nutrition education activities. Food and nutrition changes frequently discussed by women's health-care professionals included calcium, iron and folate. This finding is consistent with those of a recent study that found that pregnant women were less aware of LC $n$-3 PUFA compared to other nutrients, i.e. folate and calcium ${ }^{(27)}$.

The level of control a pregnant woman had over her actions appeared to be inversely proportional to the level of concern regarding certain issues. Health-care providers placed high importance on the issues of smoking and drug/alcohol use in pregnancy ${ }^{(30)}$ but these were rated as low levels of concern by the women. This finding may reflect that women feel they can exercise control over these matters and hence cease these behaviours when pregnant or when planning a pregnancy. Conversely, labour/birth is something women have no control over, reflected in their reported high levels of concern.

Nutrition, a central issue of concern, can be controlled to a certain degree. Hence, women who were widely informed and confident about their choices were most likely to express lower levels of concern. Women who were not informed may have low levels of concern due to lack of information or conversely express high levels of concern if aware of risks but not informed about appropriate ways to manage the risks. This issue of expressed 
concern, actual risk and ability to manage the risk is central to communicating effectively with pregnant women and should be a high priority for future research.

\section{Accessing LC n-3 PUFA information}

Health professionals were not reported to provide women with information. The women indicated they sought their own information on LC $n-3$ PUFA. The sources of information on LC $n$ - 3 PUFA most reported in this study were books/magazines. However, the accuracy of this information was not determined in the present study and it may vary and be hard to monitor. Previous studies have also reported that pregnant women sought information beyond that offered by their health-care provider $^{(34)}$. Health-care professionals providing information on LC $n$-3 PUFA are one avenue for women to gain information. Increased education of health-care providers, particularly family doctors and midwives, about LC $n$-3 PUFA and how to communicate effectively to pregnant women is clearly warranted, but so too may be the need to review information on LC $n$-3 PUFA provided by other sources such as books and magazines.

Printed education materials are free, quick and easy to read and may include places for consumers to access more information, such as information on websites ${ }^{(31,35)}$. Women in this study reported pamphlets as a preferred channel of receiving information about LC $n-3$ PUFA and mercury. However, in a related study on breast-feeding education, Guise et al. found primary care-based intervention (i.e. direct professional contact) was the most effective communication strategy and that written materials were not effective in encouraging and prolonging the duration of breast-feeding ${ }^{(36)}$. The reasons for this are unclear - perhaps the personal aspects of providing/ receiving information instil more trust or confidence in the information provided, or the nature of the topic (breastfeeding is a specific and highly personal behaviour) may lend itself to a more individually focussed approach. Whichever is the case, it suggests that what women report as a preferred channel of receiving information may not be the most effective communication strategy to bring about a behaviour change, such as changing her diet ${ }^{(31)}$. This also has implications for studies such as reported here. Is the desired outcome knowledge of nutrition (in this case LC $n$-3 PUFA, mercury and fish consumption) or the change in eating behaviours during pregnancy? At this point we do not even know whether women are receiving the appropriate information. Future research should explore more fully what impact having the information has on their dietary behaviours. The preliminary results of the present study indicate that women are likely to change their eating behaviours when equipped with appropriate information.

Public availability of written food and safety materials in Australia for pregnant women is very limited. A few resources were accessed through the course of the study and were available to pregnant women from selected organisations, including at the national level by the Food Standards Australia New Zealand and at the state level by the New South Wales Food Authority, both food regulatory agencies. However, these existing education materials mainly focused on the safety of fish consumption, providing recommendations on how often and what type of fish to have. Information about the importance and (health) benefits of LC $n-3$ PUFA and information about alternative sources of LC $n$-3 PUFA were absent. Regardless of the quality of some of this information, health-care providers appear to have limited access to such materials. This could be due to a lack of awareness of the resources, perceived lack of suitability of the material or limited provision of suitable information via their own employer, a hospital or the health department.

\section{Demographic variation/limitations}

Identifying variation due to socio-economic levels may help to segment this target population, identifying different sources of information used by subgroups of women and different ways of meeting their needs.

This study found that different levels of income and education were associated with pregnant women's knowledge. Women with a higher level of income were found to have considerably higher awareness of LC $n-3$ PUFA and the importance of eating fish during pregnancy. This is consistent with Park's and Johnson's study in 2006 reporting that women from their cohort with high income levels (24\% with $>\$ 75000 v$. $13 \%$ with $<\$ 15000$ ) have higher mercury awareness ${ }^{(37)}$. Women with higher education levels reported a higher use of the Internet and books and/or magazines than women with lower education levels. This may indicate that women with higher levels of education feel confident in relying on their own research and appraisal to gain an insight into health issues. Women with lower levels of education reported a higher incidence of not having received any information about LC $n$-3 PUFA. It appears that women with high levels of education have a higher mercury awareness ${ }^{(37)}$. Variation due to demographic factors has been commonly noted in research ${ }^{(33,38)}$. One previous study investigated sources of information of low-income pregnant women $(\leq \$ 10000-\$ 20000)(n$ 134) and identified family and friends as the more common source of information, above health-care providers ${ }^{(39)}$. However, this was not the finding of the present study, where health-care providers were identified as the main sources of information for pregnant women. In our study there were a smaller number of low-income participants ( $n$ 55), which may account for such a difference.

The results of the present study should not be considered representative of all pregnant women, particularly as those women attending private hospitals/clinics were not included in the study. Unfortunately, only a limited number of health-care facilities could be accessed in the time available for the study. The basis of the study, 
a self-administered questionnaire, may also present a limitation as it is based on self-reporting.

\section{Summary}

In summary, a large number of pregnant women reported not receiving information about LC $n$-3 PUFA during contact with the health-care system for their pregnancy. Some women reported searching themselves for this information but their knowledge was limited. Books and magazines were the main sources of LC $n-3$ PUFA information for the women in the present study. The information that women most frequently requested for provision during pregnancy was in relation to labour/birth and food and nutrition changes, and they sought this information from their family doctors. It would appear that a few government agencies are providing information on fish consumption during pregnancy, but it does not include information about LC $n-3$ PUFA, is not promoted by the health services and does not reach health-care providers or the pregnant women in their care.

\section{Acknowledgements}

The cooperation of study volunteers is gratefully acknowledged. The statistical advice provided by $\mathrm{Dr}$ Robert Clark is also gratefully acknowledged.

There were no conflicts of interest. The project was funded by the School of Health Sciences, University of Wollongong, Australia. Author contributions are as follows: B.J.M. and H.Y. came up with the original research idea. D.S carried all data collection with the assistance of D.C. D.S. analysed the data under the guidance of B.J.M. and H.Y. All authors contributed to writing of the manuscript.

\section{References}

1. Harris WS \& von Schacky C (2004) The Omega-3 Index: a new risk factor for death from coronary heart disease? Prev Med 39, 212-220.

2. Sontrop J \& Campbell M (2006) Omega-3 polyunsaturated fatty acids and depression: a review of the evidence and a methodological critique. Prev Med 42, 4-13.

3. Simopoulos AP, Leaf A \& Salem N (1999) Workshop on the essentiality of and recommended dietary intakes for omega-6 and omega-3 fatty acids. J Am Coll Nutr 18, 487-489.

4. Burdge GC, Jones AE \& Wootton SA (2002) Eicosapentaenoic and docosapentaenoic acids are the principal products of alpha-linolenic acid metabolism in young men. Br J Nutr 88, 355-363.

5. Meyer BJ, Mann NJ, Lewis JL, Milligan GC, Sinclair AJ \& Howe PRC (2003) Dietary intakes and food sources of omega- 6 and omeag-3 polyunsaturated fatty acids. Lipids 38, 391-398.

6. Howe P, Meyer B, Record S \& Baghurst K (2006) Dietary intake of long-chain [omega]-3 polyunsaturated fatty acids: contribution of meat sources. Nutrition 22, $47-53$.
7. Innis SM (2007) Dietary (n-3) fatty acids and brain development. J Nutr Educ Behav 137, 855-859.

8. Rosell MS, Lloyd-Wright Z, Appleby PN, Sanders TA, Allen NE \& Key TJ (2005) Long-chain n-3 polyunsaturated fatty acids in plasma in British meat-eating, vegetarian, and vegan men. Am J Clin Nutr 82, 327-334.

9. Lim S-Y, Hoshiba J, Moriguchi T \& Salem N (2005) N-3 fatty acid deficiency induced by a modified artificial rearing method leads to poorer performance in spatial learning tasks. Pediatr Res 58, 741-748.

10. Kitajka K, Sinclair AJ, Weisinger RS, Weisinger HS, Mathai M, Jaysooriya AP, Halver JE \& Puskás LG (2004) Effects of dietary omega-3 polyunsaturated fatty acids on brain gene expression. Proc Natl Acad Sci USA 101, 10931-10936.

11. Al MD, van Houwelingen AC \& Hornstra G (2000) Longchain polyunsaturated fatty acids, pregnancy, and pregnancy outcome. Am J Clin Nutr 71, S285.

12. Hibbeln JR \& Salem N Jr (1995) Dietary polyunsaturated fatty acids and depression: when cholesterol does not satisfy. Am J Clin Nutr 62, 1.

13. Al MD, van Houwelingen AC, Kester AD, Hasaart TH, de Jong AE \& Hornstra G (1995) Maternal essential fatty acid patterns during normal pregnancy and their relationship to the neonatal essential fatty acid status. BrJ Nutr $\mathbf{7 4}$, $55-68$.

14. National Health and Medical Research Council (2006) Nutrient Reference Values for Australia and New Zealand: Including Recommended Dietary Intakes. Canberra: Australian Government \& Ministry of Health.

15. International Society for the Study of Fatty Acids and Lipids (1999) www.issfal.org.uk (accessed May 2008).

16. Sinclair A (2007) What are the benefits of omega-3 fatty acids (omega-3s) for children - 12 months and older? Presented at Scientific Consensus Workshop: 'Omega-3 fatty acids essential nutrients for our children', Sydney, 13 August; available at www.omega3-centre.com

17. Kris-Etherton PM, Harris WS \& Appel LJ (2002) Fish consumption, fish oil, omega-3 fatty acids, and cardiovascular disease. Circulation 106, 2747-2757.

18. Better Health Channel (2005) Mercury in fish. http:// www.betterhealth.vic.gov.au/bhcv2/bhcarticles.nsf/pages/ Mercury_in_fish?OpenDocument (accessed May 2008).

19. Wikipedia http://en.wikipedia.org/wiki/methylmercury (accessed March 2008).

20. Sidhu KS (2003) Health benefits and potential risks related to consumption of fish or fish oil. Regul Toxicol Pharmacol 38, 336-344.

21. Hibbeln JR, Davis JM, Steer C, Emmett P, Rogers I, Williams C \& Golding J (2007) Maternal seafood consumption in pregnancy and neurodevelopmental outcomes in childhood (ALSPAC study): an observational cohort study. Lancet 369, 578-585.

22. Verbeke W, Sioen I, Pieniak Z, Van Camp J \& De Henauw S (2005) Consumer perception versus scientific evidence about health benefits and safety risks from fish consumption. Public Health Nutr 8, 422-429.

23. Troxell H, Anderson J, Auld G, Marx N, Harris M, Reece M \& Allen K (2005) Omega-3 for baby and me: material development for a WIC intervention to increase DHA intake during pregnancy. Matern Child Health J 9, 189-197.

24. Food Standards Australia New Zealand (2004) FSANZ Updates Advice on Mercury in Fish. Barton Act/Wellington, FSANZ.

25. Nichols P (2007) Australian seafood - best sources of omega 3 oils and safe consumption: now and in the future. Presented at Symposium on 'A focus on long chain Omega-3s', Sydney, 3 December. Hawthorn: Victoria: The Omega-3 Centre.

26. Renkert S \& Nutbeam D (2001) Opportunities to improve maternal health literacy through antenatal 
education: an exploratory study. Health Promot Int 16, 381-388.

27. Douaud C (2006) Omega-3 Least Known of pregnancy 'Big 3' Pregnancy Supplements. Washington, DC: Society for Women's Health Research.

28. Better Health Channel (2005) Pregnancy and diet. http:// www.betterhealth.vic.gov.au/bhcv2/bhcarticles.nsf/pages/ Pregnancy_and_diet?open (accessed May 2008).

29. Rubin G (1995) How Diet Can Prevent Birth Defects. New South Wales, Australia: NSW Health Department.

30. Morales S, Kendall PA, Medeiros LC, Hillers V \& Schroeder M (2004) Health care providers' attitudes toward current food safety recommendations for pregnant women. Appl Nurs Res 17, 178-186.

31. Athearn PN, Kendall PA, Hillers VV, Schroeder M, Bergmann V, Chen G \& Medeiros LC (2004) Awareness and acceptance of current food safety recommendations during pregnancy. Matern Child Health J 8, 149-162.

32. Sinikovic D, Yeatman H, Cameron D \& Meyer B (2008) Professional nutrition communication during pregnancy: a focus on long chain omega-3 polyunsaturated fatty acids. Public Health Nutr (In the Press).
33. Puder K, Rode A, Kruger M \& Gonik B (2005) Deficits in food safety knowledge during pregnancy. Am J Obstet Gynecol 193, S118-S118.

34. Aaronson LS, Mural CM \& Pfoutz SK (1988) Seeking information: where do pregnant women go? Health Educ Q 14, 335-345.

35. Begley A (2002) Barriers to good nutrient intakes during pregnancy: a qualitative analysis. Nutr Diet 59, 175-180.

36. Guise J-M, Palda V, Westhoff $\mathrm{C}$, Chan BKS, Helfand M \& Lieu TA (2003) The effectiveness of primary care-based interventions to promote breastfeeding: systematic evidence review and meta-analysis for the US Preventive Services Task Force. Ann Fam Med 1, 70-78.

37. Park S \& Johnson M (2006) Awareness of fish advisories and mercury exposure in women of childbearing age. Nutr Rev 64, 250-256.

38. Dunn CL, Pirie PL \& Hellerstedt WL (2003) The advicegiving role of female friends and relatives during pregnancy. Health Educ Res 18, 352.

39. Lewallen LP (2004) Healthy behaviors and sources of health information among low-income pregnant women. Public Health Nurs 21, 200-206. 